\title{
Value of Multidetector Computed Tomography in the Assessment of Achalasia Subtypes and Detection of Pulmonary and Thoracic Complications
}

\author{
Sanja Jovanovic ${ }^{a}$ Aleksandra Djuric-Stefanovic $^{a, c}$ Aleksandar Simić ${ }^{b, c}$ Ognjan Skrobic ${ }^{b, c}$ \\ Predrag Pesko b, c \\ ${ }^{a}$ Center of Radiology and MR, Unit of Digestive Radiology, Belgrade, Serbia; ${ }^{b}$ Department of Esophageal Surgery, \\ First Surgical University Clinic, Clinical Center of Serbia, Belgrade, Serbia; 'Faculty of Medicine, Belgrade University, \\ Belgrade, Serbia
}

\section{Significance of the Study}

- Esophageal manometry has allowed the depiction of achalasia as 3 different subtypes. So far, multidetector computed tomography has not been used as a standard imaging method in preoperative evaluation of patients with achalasia, but this study revealed that it could be a useful tool in the differentiation of achalasia subtypes, and the detection of lung and extra-esophageal thoracic complications.

\section{Keywords}

Multidetector computed tomography · Achalasia ·

Achalasia subtypes · Lung complications

\begin{abstract}
Objective: To evaluate multidetector computed tomography (MDCT) findings in patients with achalasia, to assess its role in differentiating subtypes in detecting lung involvement and extra-esophageal thoracic complications. Subjects and Methods: This clinical retrospective study included 51 patients with manometrically confirmed achalasia who underwent chest X-ray and MDCT in diagnostic work-up. Esophageal wall thickness and morphology, luminal dilatation, lung changes, and extra-esophageal manifestations were analyzed on MDCT by 2 readers. Wilcoxon, Kruskal-Wallis and Mann-Whitney test were used for assessing the differences among the achalasia subtypes, and intra-class correlation coefficients (ICC) assessing the inter-observer agreement between the measurements of 2 readers. Results:
\end{abstract}

Fourteen (27.5\%) patients had achalasia subtype I, 21 (60.8\%) had subtype II while 6 (11.8\%) had subtype III. Esophageal wall thickness of the esophageal body (EB) and distal esophageal segment (DES) as well as nodular/lobulated appearance of DES were found significantly more often in subtype III ( $p=0.024, p<0.001, p=0.009$, respectively). Esophageal dilatation gradually decreased from subtype I to III ( $p=$ $0.006)$. Chest X-ray revealed lung changes in $9(17 \%)$ and MDCT in $21(41 \%)$ patients ( $p=0.001)$, most frequently in subtype I, with predominance of ground-glass opacities. Tracheal/carinal compression was detected in 27 (52.9\%) and left atrial compression in 17 (33.3\%) patients. Excellent interobserver agreement was observed in measuring the EB and DES wall thickness, and diameter of EB (ICC 0.829, 0.901, and 0.922). Conclusion: MDCT is a useful tool for detecting lung and extra-esophageal thoracic complications in patients with achalasia, and could be a valuable additional imaging modality in the differentiation of achalasia subtypes.

(c) 2019 The Author(s)

Published by S. Karger AG, Basel

\begin{tabular}{|c|c|c|}
\hline KARGER & $\begin{array}{l}\text { (c) } 2019 \text { The Author(s) } \\
\text { Published by S. Karger AG, Basel }\end{array}$ & $\begin{array}{l}\text { Karger } \\
\text { Open access }\end{array}$ \\
\hline $\begin{array}{l}\text { E-Mail k } \\
\text { www.ka }\end{array}$ & $\begin{array}{l}\text { This is an Open Access article licensed } \\
\text { Attribution-NonCommercial- } 4.0 \text { Inte } \\
\text { (http://www.karger.com/Services/Ope } \\
\text { the online version of the article only. } L \\
\text { mercial purposes requires written per }\end{array}$ & $\begin{array}{l}\text { the Creative Commons } \\
\text { al License (CC BY-NC) } \\
\text { sLicense), applicable to } \\
\text { d distribution for com- }\end{array}$ \\
\hline
\end{tabular}

Sanja Jovanovic

Center for Radiology and MRI

Clinical Center of Serbia, Pasterova 2

RS-11000 Belgrade (Serbia)

E-Mail dr.sanja.jovanovic@gmail.com 


\section{Introduction}

Achalasia is a primary esophageal motility disorder of the unknown origin characterized by the absence of esophageal peristalsis and impaired relaxation of the lower esophageal sphincter (LES), clinically presenting as dysphagia and regurgitation. The technical progress in functional esophageal diagnostics in the last 2 decades has significantly improved our understanding of the underlying mechanisms, as well as its diagnosis and treatment [1].

The initial diagnostic step when achalasia is suspected in patients with dysphagia is usually barium esophagography, assessing the degree of esophageal dilatation, axis of the esophagus, esophageal emptying, opening of the LES and the probable presence of the associated epiphrenic diverticulum [2]. Upper gastrointestinal endoscopy may be inconclusive in patients with achalasia. Esophageal manometry has been a gold standard for diagnosis of achalasia, demonstrating the main features of the disease in the form of failure of peristalsis and impaired relaxation of LES [3]. The advent of high-resolution esophageal manometry has allowed further depiction of achalasia in 3 different subtypes, differentiated by abnormalities in contractile and pressurization patterns of the esophageal body (EB) [4]. Nevertheless, subsequent studies have shown that these forms of achalasia can also be revealed on the basis of conventional manometry (CM) esophageal [5]. Today, the foremost importance of classification of achalasia in 3 different subtypes is in the rational choice of best initial therapeutic approach and prediction of the treatment outcome [6]. The advent of highly specific functional diagnostics such as high-resolution esophageal manometry has brought important new perspectives into the understanding and management of achalasia, although some radiological confusion including multidetector computed tomography (MDCT) patterns still persists [6-8]. Contemporary understanding of achalasia defines it as a heterogeneous disease that can be categorized into 3 distinct subtypes based on manometric findings principally depending on esophageal pressurization [4-8]. Based on previous investigations, the most common form of achalasia is subtype II [9-11]. To date, most of the studies have shown that patients with achalasia subtype II have the best treatment outcomes, whereas those with subtype I and especially subtype III have less favorable outcomes [4-7].

Even though the prevalence of pulmonary complications caused by chronic (micro) aspiration in patients with achalasia is high, reaching the incidence of $>50 \%$, in most instances the only diagnostic tool used is the plain chest X-ray, with a very low sensitivity and specificity [12]. In contrast, correct detection of pulmonary complications can be of outmost importance in the proper preoperative workup and ultimate treatment of patients with achalasia [13]. So far, MDCT has not been used as a standard imaging method in preoperative evaluation of patients with achalasia [14]. Today, the most common indication for MDCT in patients with achalasia is differentiating the primary form from submucosal infiltration of the distal esophagus and esophagogastric junction (EGJ), mainly caused by neoplasms, which is commonly referred to as pseudoachalasia [14-16].

With the increasing use of MDCT in everyday clinical practice, it is becoming apparent that this imaging test may have a significant role in the accurate evaluation of patients with achalasia [12, 13, 17-22]. To our knowledge, no comprehensive study has analyzed the significance of MDCT in the differential diagnosis of achalasia subtypes and on their impact on pulmonary complications. The purpose of our study was to evaluate MDCT findings and assess the utility of MDCT in the differentiation of achalasia subtypes by assessing esophageal wall morphology, with special emphasis on extraesophageal lung and thoracic complications.

\section{Subjects and Methods}

The study population consisted of 51 consecutive, previously untreated patients who were diagnosed with achalasia by manometry. It was designed as a single-institution-based clinical retrospective study and was a part of larger prospective study on the prediction of early and late success of surgery in 3 subtypes of achalasia by using different radiologic methods. This study was approved by the Hospital Board and Ethics Committee, and written informed consent was obtained from all subjects before enrollment. Based on the incidence and prevalence of achalasia from the current literature and $95 \% \mathrm{CI}$, the minimum number of patients to be studied is reported to be $35[8,23,24]$. An inclusion criterion was achalasia diagnosed by CM, while exclusion criteria were related to the presence of pseudoachalasia caused by neoplasm at the level of the EGJ. Fifty-two patients were included, and one excluded due to co-existing EGJ neoplasm. All patients underwent surgical laparoscopic Heller myotomy in our Clinic between October 2015 and December 2016.

Based on CM findings, achalasia was divided into 3 subtypes according to the following criteria [4-6]:

Achalasia subtype I: EB contraction with maximal pressure below $30 \mathrm{~mm} \mathrm{Hg}$;

Achalasia subtype II: EB waves with pressure above $30 \mathrm{~mm} \mathrm{Hg}$ in at least $20 \%$ of swallows;

Achalasia subtype III: long-lasting, high pressure spastic esophageal contraction in $\geq 20 \%$ of swallows. 

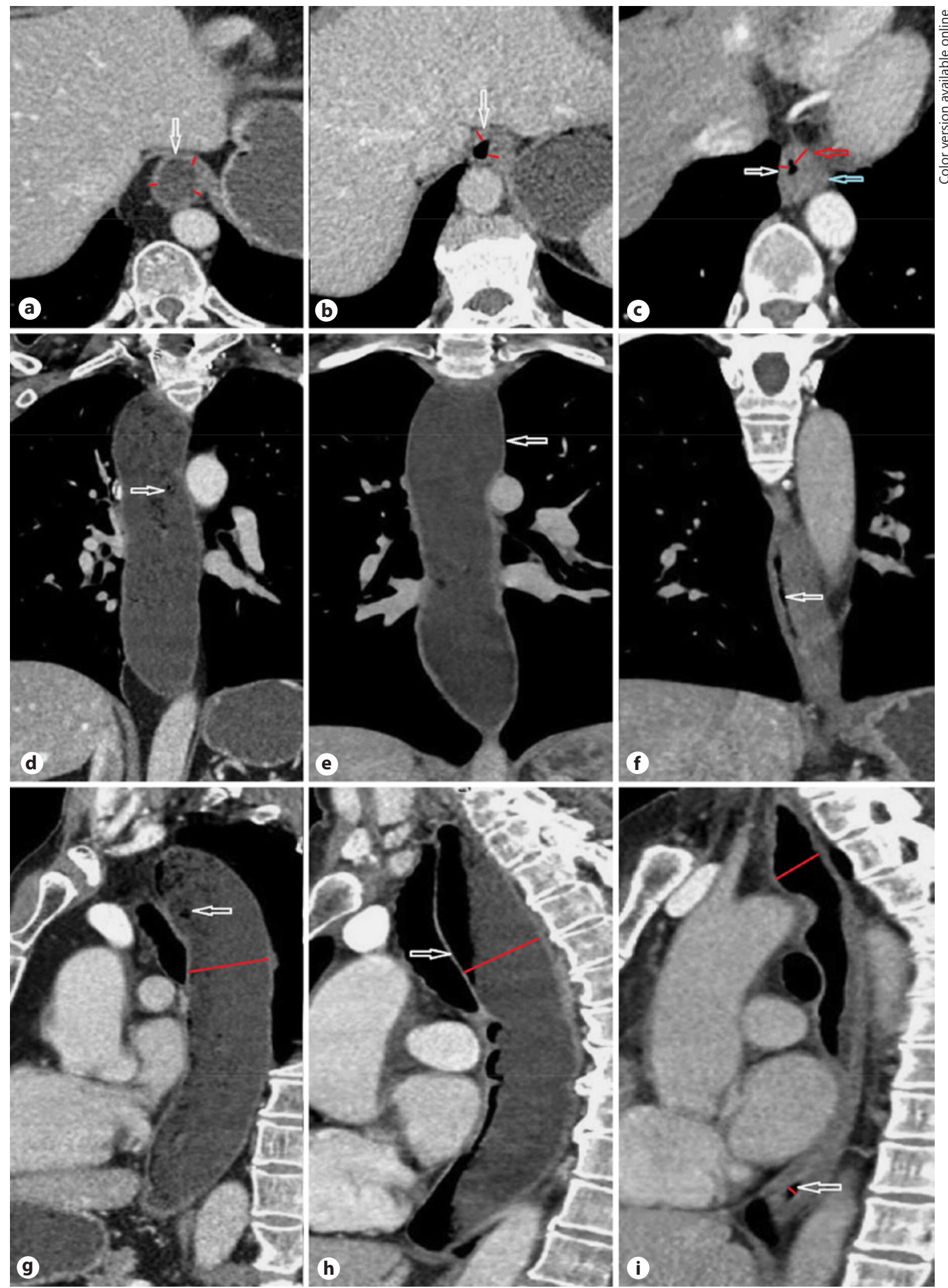

Fig. 1. MDCT performed in the axial (1st row) plane at the level of DES, coronal (2nd row) and sagittal (3rd row) plane, according to achalasia subtypes (a, d, g: subtype I; b, e, h: subtype II and $\mathbf{c}, \mathbf{f}$, i: subtype III). Slight and symmetrical wall thickening (white arrow, red line) without lobulated margins (a), moderate and symmetric wall thickening (white arrow, red line) without lobulated (blue ar- row) margins (b), remarkable (white arrow) and asymmetric (red arrow, red line) wall thickening (c). Remarkably and uniformly dilatation of full-length EB (red line) with liquid and gas contents (d, g, white arrow), moderate and less uniformly dilatation (red line) of EB with liquid content (e, h, white arrow), discrete dilatation (white arrow) of $\mathrm{EB}(\mathbf{f}, \mathbf{i})$. 
Table 1. MDCT presentation of pulmonary and extra-esophageal thoracic manifestations in patients with three different subtypes of achalasia

\begin{tabular}{|c|c|c|c|c|c|}
\hline $\begin{array}{l}\text { Pulmonary and extra-esophageal } \\
\text { thoracic manifestations (MDCT) }\end{array}$ & $\begin{array}{l}\text { Subtype I } \\
(n=14), n(\%)\end{array}$ & $\begin{array}{l}\text { Subtype II } \\
(n=31), n(\%)\end{array}$ & $\begin{array}{l}\text { Subtype III } \\
(n=6), n(\%)\end{array}$ & $p$ value ${ }^{\S}$ & $\begin{array}{l}\text { Total } \\
(n=51), n(\%)\end{array}$ \\
\hline Ground-glass opacities & $9(64.3)$ & $9(29.0)$ & 0 & \multirow[t]{3}{*}{0.012} & $18(35.3)$ \\
\hline Segmental consolidation & $1(7.1)$ & $1(3.2)$ & 0 & & $4(7.8)$ \\
\hline Lobar consolidation & $1(7.1)$ & 0 & 0 & & $1(2)$ \\
\hline Tracheal/carinal compression & $8(57.1)$ & $16(51.6)$ & $3(50.0)$ & 0.933 & $27(52.9)$ \\
\hline Decreased transparency & $6(42.8)$ & $3(9.7)$ & 0 & \multirow[t]{3}{*}{0.296} & $9(17.6)$ \\
\hline Consolidation & $3(21.4)$ & 0 & 0 & & $3(5.86)$ \\
\hline Collapse & 0 & $1(3.3)$ & 0 & & $1(1.95)$ \\
\hline
\end{tabular}

${ }^{\S}$ Kruskal-Wallis test.

MDCT, multidetector computed tomography.

In preoperative workup, after the $\mathrm{CM}$, the plain chest $\mathrm{X}$-ray and MDCT examination of the thorax and abdomen were done in every patient. The indication for MDCT examination was dysphagia.

MDCT examinations were done at least 2 days after the manometry by using a 64-detector scanner (Aquilion CXL, Toshiba; in helical-mode, $0.5 \mathrm{~mm}$ section thickness, $120 \mathrm{kV}, 120-750 \mathrm{mAs}$ in tube current modulation mode, $0.5 \mathrm{~s}$ rotation time, $26.5 \mathrm{~mm} / \mathrm{s}$ table speed, $32-\mathrm{cm}$ scan field of view). The MDCT images were reconstructed in axial, sagittal, and coronal planes, with reconstructed slice thickness of $1 \mathrm{~mm}$. The soft tissue and lung tissue algorithm were used for the analysis. Oral (150 mL of clear water) and intravenous contrast agents were given, and CT scanning was performed in a single portal venous contrast-enhanced phase. A standard dose of $80 \mathrm{~mL}$ iopromide (Ultravist 370, Bayer-Schering, Leverkusen, Germany) was employed for intravenous administration of contrast media.

Plain chest X-rays and MDCT exams were analyzed by 2 general radiologists (S.J. and A.D.-S. with 4- and 18-year experience in abdominal and general radiology), through consensus. Readers were blinded for CM findings of achalasia subtype.

Plain chest X-rays were analyzed to detect decreased transparency of lung parenchyma, consolidation, or lung collapse.

In the MDCT examination, the esophageal wall thickness was measured in the widest part of mid-thoracic esophagus, at the level of carina $\mathrm{EB}$, and $3 \mathrm{~cm}$ above the cardia (distal esophageal segment, DES) in the axial plane, using electronic caliper (Fig. 1a-f). MDCT morphology of DES wall thickening was divided into 2 patterns: smooth and nodular/lobulated, which was analyzed in the axial plane (Fig. 1a-c). Esophageal dilatation was measured as maximal anterior-posterior (AP) diameter of the EB (Fig. 1g-i). Measurements of both readers were compared in order to assess the inter-observer agreement, and mean values from measurements by both readers' were obtained and statistically analyzed.

In the evaluation of lung parenchyma, MDCT images were analyzed to determine the existence of ill-defined opacities (ground-glass, tree-in-bud), presence and distribution of consolidation, and lung collapse (Table 1; Fig. 2a-c). In addition, the extra-esophageal thoracic manifestations of achalasia (compression of trachea or carina and compression of left atrium) were visually searched (Fig. 2d-f) [19-22].

\section{Statistical Analysis}

The Shapiro-Wilk test was used for the assessment of normal statistical data distribution. Descriptive statistical methods used were the measures of the central tendency (arithmetic mean \pm SD or median). For assessing the inter-observer agreement between the measurements of 2 readers, the intra-class correlation coefficients were calculated. Kruskal-Wallis, Mann-Whitney and Wilcoxon signed rank test were used for assessing the differences among achalasia subtypes. Kappa test $(\mathrm{K})$ was used to assess the agreement between the plain chest radiography and MDCT in detectability of lung parenchyma pathological changes. Statistical data analysis was performed using the IBM SPSS Statistics 22.0 software (IBM Corporation, Armonk, NY, USA).

\section{Results}

\section{Study Population}

Study group consisted of 51 patients, out of whom 31 were females, with the mean age of $53.7 \pm 16.5$ years (range 20-87). An average preoperative duration of symptoms was $4.2 \pm 2.1$ years. In 14 patients $(27.5 \%)$, achalasia subtype I was diagnosed by using the manometry, in $31(60.8 \%)$ subtype II, and in 6 patients $(11.8 \%)$ subtype III.

\section{Esophageal Wall Morphology and Luminal Diameter}

Median values of esophageal wall thickness of EB and DES, and esophageal dilatation (maximal AP diameter of EB) and differences in 3 achalasia subtypes were presented in Table 2.

Differences of median values of the esophageal wall thickness of EB were tested by using the Mann-Whitney test and reached statistical significance between the sub- 

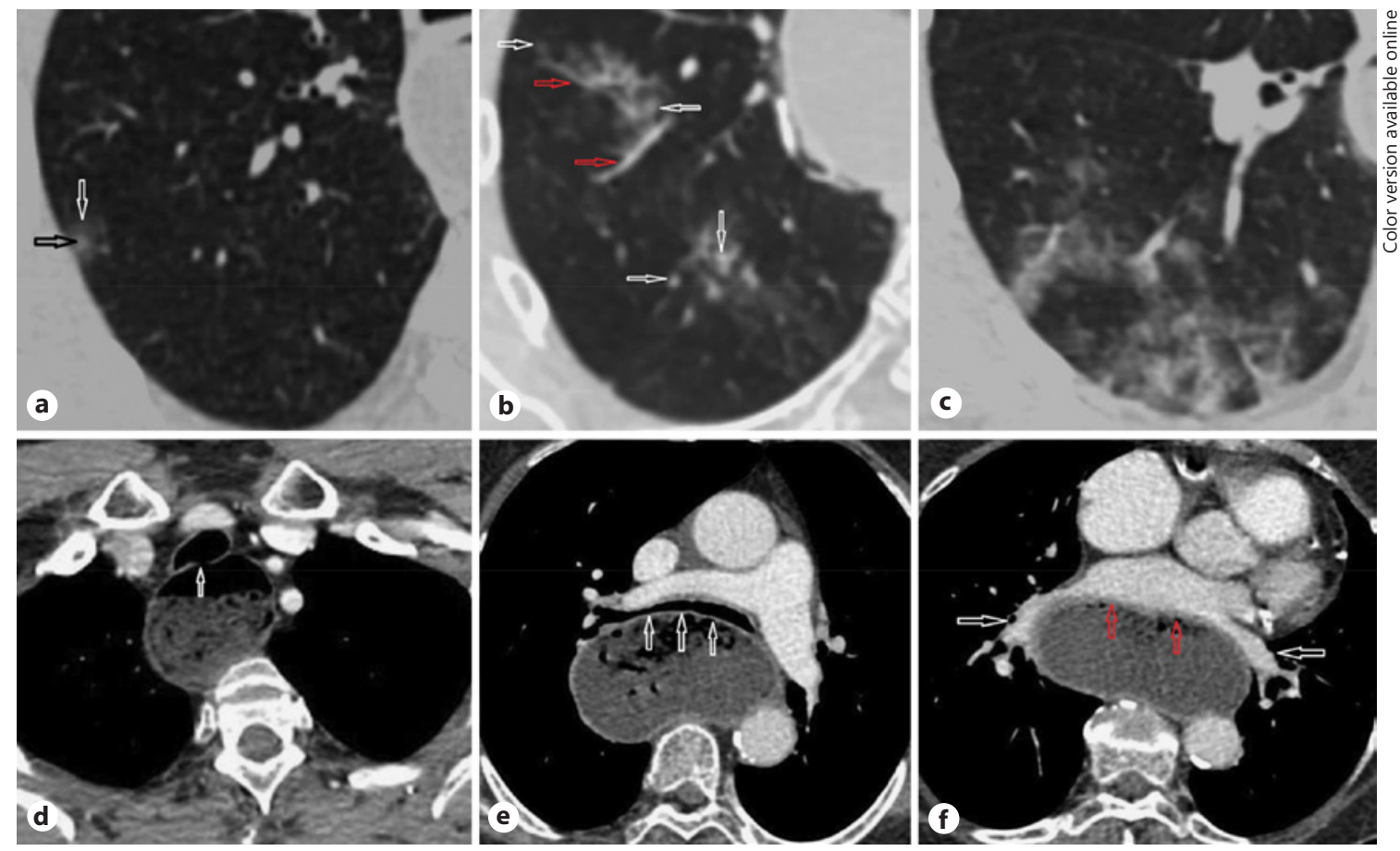

Fig. 2. Pulmonary complications (1st row) and extra-esophageal thoracic manifestations of achalasia (2nd row). MDCT performed in the axial plane: sub-pleural micro-nodular pattern (black arrow) with surrounding "ground-glass" (white arrow) opacities (a), multiple intra-parenchymal micro-nodular patterns (white arrow) with striped (red arrow) and irregular samples mimicking "tree-in-bud" presentation (b), "ground-glass" consolidation of lung parenchyma (c). Compression of the posterior tracheal wall (white arrow) with its displacement anteriorly (d), compression of the carina (white arrow; e), and compression of the left atrium (red arrow) and pulmonary veins (white arrow), by dilated esophagus (f). types I and III ( $p=0.013)$, and subtypes II and III ( $p=$ 0.010 ), while at the level of DES significant difference in wall thickness was proven between the subtypes I and II ( $p \otimes 0.001)$, subtypes I and III ( $p=0.004)$, and subtypes II and III $(p=0.015)$. Significant difference in maximal AP diameter of the dilated EB was proven between the subtypes I and III ( $p=0.006)$, and subtypes II and III ( $p=$ $0.019)$, while between the subtypes I and II, it did not reach the statistical significance $(p=0.059)$.

Nodular and/or lobulated presentation of DES was detected in 15 patients $(29.4 \%)$, with predominance in subtype III (5 of 6 patients, $83.3 \%$ ), showing statistically significant differences among the 3 subtypes $(p=0.009)$.

Typical MDCT appearances of achalasia subtype I, II, and III were presented in (Fig. 1a-i).

\section{Analysis of Pulmonary and Extra Esophageal Thoracic Manifestations}

The chest X-ray examination revealed lung parenchymal pathological patterns in $9(17.6 \%)$ patients. All of them manifested decreased transparency, which was pre- sented as patchy, fuzzy, and less demarcated parenchymal shadings. Consolidation was present in 3 , while the lung collapse was found in 1 patient (Table 1). Decreased transparency and segmental consolidation were predominant in subtype I (11.7\%), whereas the lung collapse was present in one patient in subtype II, but differences among groups were not statistically significant (Table 1).

Using MDCT the lung parenchymal changes were noted in $21(41.2 \%)$ patients, which is significantly more frequent than by using the plain chest X-ray in the same patients $(Z=-3.464, p=0.001)$. Moderate agreement was proven by using the Kappa test between the plain chest radiography and MDCT examination in detecting the lung parenchymal pathological changes $(\mathrm{K}=0.469, p<$ $0.001)$. In summary, 12 patients in the whole study group (23.5\%) had false negative findings in the plain chest radiography, since lung parenchyma changes were detected in their CT examinations, while in the remaining 9 patients (17.6\%) with visible lung parenchyma pathological changes in the plain chest radiography, those findings were confirmed by CT (Table 1). 
Table 2. Esophageal wall thickness and esophageal dilatation measured at different levels in the axial plane, according to achalasia subtypes

\begin{tabular}{|c|c|c|c|c|}
\hline & $\begin{array}{l}\text { Subtype I }(n=14) \\
\text { median }(\min -\max )\end{array}$ & $\begin{array}{l}\text { Subtype II }(n=31) \\
\text { median }(\min -\max )\end{array}$ & $\begin{array}{l}\text { Subtype III }(n=6) \\
\text { median }(\min -\max )\end{array}$ & $p$ value $^{\S}$ \\
\hline \multicolumn{5}{|l|}{ Wall thickness, mm } \\
\hline $\mathrm{EB}$ & $3(2-6)$ & $3(1-8)$ & $6(3-8)$ & 0.024 \\
\hline DES & $6(3-8)$ & $8(5-13)$ & $10(6-10)$ & $<0.001$ \\
\hline \multicolumn{5}{|l|}{ Dilatation, $\mathrm{mm}$} \\
\hline AP diameter EB & 45 (17-95) & $37(15-64)$ & $26(13-29)$ & 0.006 \\
\hline
\end{tabular}

Lung manifestations were detected in MDCT examinations of 11 patients (78.6\%) who had achalasia subtype I, in $10(47.6 \%)$ with the subtype II, and no one with the subtype III $(p=0.001)$. The ground-glass opacities were dominant patterns seen in 18 patients, tree-in-bud in 4 , segmental consolidation in 2, and lobar consolidation in 1 patient. Ground-glass opacities and tree-in-bud were principal findings in subtype I (64.3\%) and subtype II (29\%; Table 1).

Extra esophageal thoracic manifestations of achalasia such as compression of trachea and/or carina by dilated esophagus were detected in 27 patients (52.9\%), while compression of the left atrium was noted in 18 patients $(35.3 \%)$, predominantly in type I (Table 1; Fig. $2 \mathrm{~d}-\mathrm{f}$ ).

\section{Inter-Observer Agreement of Measurements}

Intra-class correlation coefficients between the measurements of 2 readers of EB and DES wall thickness, and diameter of EB were $0.829,0.901$, and 0.922 , respectively, which had shown excellent inter-observer agreement $(p<0.001)$.

\section{Discussion}

Similar distribution of frequencies of 3 subtypes of achalasia as in our patient cohort was reported in other published series $[9-12,25]$. Spastic achalasia, which revealed poor treatment success, was the rarest subtype comprised of only 6 patients in our patient-group, which is in agreement with the findings of other studies from different countries [9-11,25].

Regardless of the degree of luminal distention, it was reported that normal esophageal wall thickness had ranged between 2 and $5 \mathrm{~mm}$, as described by Xia et al.
[26], who observed different values of esophageal wall thickness depending on esophageal dilatation or contraction. The first published study on MDCT evaluation of patients with achalasia concluded that wall thickening was atypical with moderate mean luminal dilatation of $43.5 \mathrm{~mm}$ at the level of carina [21]. To our knowledge, in the current literature, there is no study indicating the exact values of the wall thickening in patients with esophageal motility disorders [26], except for the investigation of Goldberg et al. [27], which included a small number of patients with esophageal spasm. Our investigation revealed that the majority of patients with achalasia had thickened esophageal wall in the distal segment; in addition, the patients with subtype III of achalasia had significantly higher median value of esophageal wall thickness of the EB compared to other 2 subtypes. Moreover, the esophageal wall thickness of both EB and DES was found to gradually increase from subtype I to subtype III, which could have further implications on role of MDCT in differentiating achalasia subtypes. Our data are in concordance with Mittal et al. [28] who showed that muscle thickness in patients with achalasia was largest in subtype III, while the subtype II had thicker muscle layer than subtype I by evaluating the ultrasonographic cross-sectional area and wall thickness of the esophageal muscle by using the endoscopic esophageal ultrasonography. The differences in wall thickness could be accurately assessed only on the basis of whether the luminal dilatation had been similar for the 3 subgroups. Accordingly, lesser dilatation of subtype III could influence the differences in wall thickness measured in the MDCT scans, higher for spastic achalasia than the others. Given that we measured the thickest esophageal wall in patients with the subtype III and that these patients had the highest intraluminal pressure confirmed by $\mathrm{CM}$, a similar degree of dilatation 
between this and other 2 subtypes could not be expected. One potential explanation could be that the augmented EB contractility in patients with spastic achalasia was associated with an increase in the circular and longitudinal muscle thickness, as well as in axial shortening of the esophagus [29].

Previous studies revealed that esophageal wall thickness $>10 \mathrm{~mm}$ had been indicated by the neoplasm, while mild and symmetric distal esophageal wall thickening suggested the diagnosis of achalasia [15]. Furthermore, the increased wall thickness over $5 \mathrm{~mm}$ with nodular and/ or lobulated asymmetric pattern of the DES was believed to be highly suggestive of pseudoachalasia [16]. Our study documented a high incidence of nodular and/or lobulated, asymmetrically narrowed segment of the distal esophageal wall, with its predominant appearance in subtype III. This appearance could be explained by spastic contractions of the esophagus in subtype III recorded by MDCT examination. Therefore, our findings indicate that a nodular and/or lobulated pattern and thickened esophageal wall might not be always the sign of neoplasm and should be analyzed with regard to achalasia subtype.

Marked esophageal dilatation was commonly seen in patients with achalasia subtype I and II, combined with various amounts of liquid and food residue. In our study, the widest median diameter of $45 \mathrm{~mm}$ was noted in patients with achalasia subtype I, which is in accordance with the previous studies, confirming that their esophageal width was larger than in other subtypes $[5,25]$. The lowest median esophageal diameter was observed in patients with the subtype III of achalasia, probably due to non-progressive EB pressurization, limiting the further widening of the lumen [25].

Although earlier reports indicated that patients with achalasia experienced respiratory symptoms in about $10 \%$ of cases, in recent studies their prevalence has been observed in over $40 \%$ of cases [7, 17]. Makharia et al. [17] even showed that $53 \%$ of achalasia patients had various degrees of structural or functional lung abnormalities, while our study reported $41.2 \%$ of patients with structural lung pathological findings found on MDCT. The mechanisms of lung involvement in achalasia patients include extrinsic compression of the tracheobronchial tree by the dilated esophagus as well as repeated micro aspirations. It has also been noted that the incidence of the structural lung abnormalities is increasing with the enlargement of esophageal width $[18,19]$, which is in agreement with our results.

In general, chest X-ray as a basic imaging method in preoperative assessment of patients with achalasia has a very low sensitivity and specificity for pulmonary changes, which was documented in our study; only $17.6 \%$ of patients were presented with lung parenchymal changes detected on chest X-ray in comparison to $41.2 \%$ detected by MDCT. MDCT recognized lung abnormalities mostly as patchy unilateral or bilateral ground-glass opacities of the increased attenuation with a tree-in-bud appearance, centrilobular nodules, and poorly marginated acinar areas of the increased attenuation [17]. The most common pulmonary pattern seen by MDCT in our study population was ground-glass opacities, probably as a sign of interstitial pneumonitis, which developed after the acute phase of aspiration [23]. This finding was most common in achalasia subtype I (64.3\%) and significantly less in subtype II (29\%). The second most common pulmonary MDCT finding in our study was tree-in-bud, implicating luminal impaction within bronchioles that is, aspiration bronchiolitis [22], also mainly manifested in subtype I (21.4\%). Lung consolidations (segmental or lobar) were most common in achalasia subtype I, and observed in $10 \%$ of these patients. In achalasia patients, these findings most often implicate pneumonia and demand specific preoperative therapeutic workup. Contrary to these findings, patients with subtype III did not have any visible changes in lung parenchyma.

Even though the compression of the respiratory tract and consecutive segmental atelectasis may remain asymptomatic, a significant number of patients with achalasia has marked compression of the airways and heart. Esophageal dilatation can lead to tracheal compression, and in some conditions even respiratory distress, while compression of the left atrium, usually provoking mild chest discomfort, may result in hemodynamic instability $[19,20]$. In our study, tracheal compression at the level of carina was seen in $52.9 \%$ and in the left atrium in $35.3 \%$ of all patients. Both entities were most commonly seen in achalasia subtype I, which was an expected outcome due to the largest esophageal diameter within this subgroup of patients. Although no left atrial compression was noted in patients with subtype III, the compression of the carina due to dilated and diffusely thickened esophagus was seen in $50 \%$ of cases.

The current study has several limitations. First, patients with achalasia are often unable to drink a sufficient amount of clear water before the MDCT examination. This could result in insufficient luminal distension and inadequate evaluation of the esophageal wall thickness. Second, our study group included only 6 patients with achalasia subtype III, which might diminish the power of statistical conclusions. Further investigation is needed for evaluating our 
findings of the characteristic MDCT presentation of this subtype of achalasia. Another limitation was the lack of analysis of inter-observer agreement by using the kappa score to assess lung changes on X-ray and MDCT scans.

\section{Conclusion}

Chest X-ray has failed to provide sufficient data about lung parenchymal involvement and tracheal and/or cardiac compression in patients with achalasia, warranting the role of MDCT as useful clinical and diagnostic tool.
This may be of utmost importance in patients with achalasia subtype I, in whom significant involvement of lung comorbidities has been shown. The recognition of these changes would facilitate proper preoperative workup and initial clinical management. According to presented results, which need further external validation on larger sample sites, it can be concluded that MDCT could be an additional valuable imaging modality in differentiating the 3 subtypes of achalasia according to wall thickness, luminal dilatation, and esophageal morphology, with the emphasis on its advantage in detecting pulmonary and extra esophageal thoracic complications.

\section{References}

1 Park W, Vaezi MF. Etiology and pathogenesis of achalasia: the current understanding. Am J Gastroenterol. 2005 Jun;100(6):140414.

2 Vaezi MF, Richter JE; American College of Gastroenterology Practice Parameter Committee. Diagnosis and management of achalasia. Am J Gastroenterol. 1999 Dec;94(12): 3406-12.

3 Ayazi S, Hagen JA, Zehetner J, Ross O, Wu C, Oezcelik A, et al. The value of high-resolution manometry in the assessment of the resting characteristics of the lower esophageal sphincter. J Gastrointest Surg. 2009 Dec; 13(12):2113-20.

4 Pandolfino JE, Kwiatek MA, Nealis T, Bulsiewicz W, Post J, Kahrilas PJ. Achalasia: a new clinically relevant classification by high-resolution manometry. Gastroenterology. 2008 Nov;135(5):1526-33.

5 Salvador R, Costantini M, Zaninotto G, Morbin T, Rizzetto C, Zanatta L, et al. The preoperative manometric pattern predicts the outcome of surgical treatment for esophageal achalasia. J Gastrointest Surg. 2010 Nov;14(11):1635-45.

6 Boeckxstaens GE, Zaninotto G, Richter JE. Achalasia. Lancet. 2014 Jan;383(9911):8393.

7 Crespin OM, Tatum RP, Xiao K, Martin AV, Khandelwal S, Pellegrini CA, et al. The relationship between manometric subtype and outcomes of surgical treatment for patients with achalasia : Achalasia: manometric subtypes. Surg Endosc. 2017 Dec;31(12):506675.

8 Patel DA, Lappas BM, Vaezi MF. An overview of achalasia and its subtypes. Gastroenterol Hepatol (N Y). 2017 Jul;13(7):411-21.

9 Roman S, Zerbib F, Quenehervé L, Clermidy H, Varannes SB, Mion F. The Chicago classification for achalasia in a French multicentric cohort. Dig Liver Dis. 2012 Dec;44(12): 976-80.
10 Yamashita H, Ashida K, Fukuchi T, Nagatani $\mathrm{Y}$, Koga H, Senda K, et al. Predictive factors associated with the success of pneumatic dilatation in Japanese patients with primary achalasia: a study using high-resolution manometry. Digestion. 2013;87(1):23-8.

11 Min M, Peng LH, Yang YS, Hou XH, Guo RB, Wang WF, et al. Characteristics of achalasia subtypes in untreated Chinese patients: a high-resolution manometry study. J Dig Dis. 2012 Oct;13(10):504-9.

12 Sinan H, Tatum RP, Soares RV, Martin AV, Pellegrini CA, Oelschlager BK. Prevalence of respiratory symptoms in patients with achalasia. Dis Esophagus. 2011 May;24(4):224-8.

13 Layton J, Ward PW, Miller DW, Roan RM. Acute respiratory failure secondary to esophageal dilation from undiagnosed achalasia. A A Case Rep. 2014 Sep;3(5):65-7.

14 Ba-Ssalamah A, Zacherl J, Noebauer-Huhmann IM, Uffmann M, Matzek WK, Pinker $\mathrm{K}$, et al. Dedicated multi-detector CT of the esophagus: spectrum of diseases. Abdom Imaging. 2009 Jan-Feb;34(1):3-18.

15 Carter M, Deckmann RC, Smith RC, Burrell MI, Traube M. Differentiation of achalasia from pseudoachalasia by computed tomography. Am J Gastroenterol. 1997 Apr;92(4): 624-8.

16 Licurse MY, Levine MS, Torigian DA, Barbosa EM Jr. Utility of chest CT for differentiating primary and secondary achalasia. Clin Radiol. 2014 Oct;69(10):1019-26.

17 Makharia GK, Seith A, Sharma SK, Sinha A, Goswami P, Aggarwal A, et al. Structural and functional abnormalities in lungs in patients with achalasia. Neurogastroenterol Motil. 2009 Jun;21(6):603-8.

18 Miyamoto S, Konda Y, Matsui M, Sawada K, Ikeda K, Watanabe N, et al. Acute airway obstruction in a patient with achalasia. Intern Med. 2011;50(20):2333-6.

19 Doshi AH, Aw J, Costa F, Cohen L, Som PM. Cervical tracheal compression in a patient with achalasia: an uncommon event. AJNR Am J Neuroradiol. 2009 Apr;30(4):813-4.
20 Felix AD, Quintes AC, Andrade de Sá G, Adachi MR, Fittipaldi V, Paula Dos Reis Velloso Siciliano A. Left atrial compression secondary to massive esophageal dilatation in a patient with idiopathic achalasia. CASE (Phila). 2018 Aug;2(6):285-92.

21 Rabushka LS, Fishman EK, Kuhlman JE. CT evaluation of achalasia. J Comput Assist Tomogr. 1991 May-Jun;15(3):434-9.

22 Giménez A, Franquet T, Erasmus JJ, Martínez S, Estrada P. Thoracic complications of esophageal disorders. Radiographics. 2002 Oct;22(Spec No suppl_1):S247-58.

23 Eng J. Sample size estimation: how many individuals should be studied? Radiology. 2003 May;227(2):309-13.

24 Sadowski DC, Ackah F, Jiang B, Svenson LW. Achalasia: incidence, prevalence and survival. A population-based study. Neurogastroenterol Motil. 2010 Sep;22(9):e256-61.

25 Khan MQ, AlQaraawi A, Al-Sohaibani F, AlKahtani K, Al-Ashgar HI. Clinical, endoscopic, and radiologic features of three subtypes of achalasia, classified using high-resolution manometry. Saudi J Gastroenterol. 2015 May-Jun;21(3):152-7.

26 Xia F, Mao J, Ding J, Yang H. Observation of normal appearance and wall thickness of esophagus on CT images. Eur J Radiol. 2009 Dec;72(3):406-11.

27 Goldberg MF, Levine MS, Torigian DA. Diffuse esophageal spasm: CT findings in seven patients. AJR Am J Roentgenol. 2008 Sep; 191(3):758-63.

28 Mittal RK, Hong SJ, Bhargava V. Longitudinal muscle dysfunction in achalasia esophagus and its relevance. J Neurogastroenterol Motil. 2013 Apr;19(2):126-36.

29 Kim TH, Patel N, Ledgerwood-Lee M, Mittal RK. Esophageal contractions in type 3 achalasia esophagus: simultaneous or peristaltic? Am J Physiol Gastrointest Liver Physiol. 2016 May;310(9):G689-95.

Jovanovic/Djuric-Stefanovic/Simić/ Skrobic/Pesko 